\title{
The Design of Environment Online Data Acquisition System of Hongze Lake Based on GPRS
}

\author{
Dai Junfeng ${ }^{1,2, a}$, Fu Li-hui ${ }^{3, b}$,Wang Li-fei ${ }^{3, c}$ \\ ${ }^{1}$ Faculty of Electronic Information Engineering,Huaiyin Institute of Technology, Jiangsu \\ Huaian,223003, China \\ ${ }^{2}$ College of Automation Engineering, Nanjing University of Aeronautics and Astronautics,Nanjing \\ 210016,China \\ ${ }^{3}$ Faculty of Automation, Huaiyin Institute of Technology,Jiangsu Huaian,223003,China

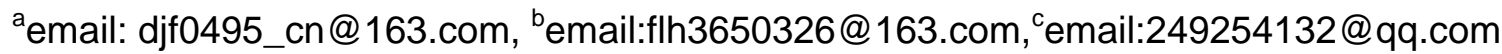

Keywords: water quality monitoring ;GPRS;NiosIl system;the paperless recorder;remote data transmission

\begin{abstract}
The environment online data acquisition system of Hongze Lake based on GPRS is designed,which based on detecting the temperature, $\mathrm{pH}$ and dissolved oxygen of water in Hongze Lake,using SOPC technology in a FPGA chip to construct a NiosII microprocessor and its peripheral interface circuit,accordingly forming a custom microprocessor system consistent with the task demand of system.After the NiosII microprocessor convert temperature,pH and dissolved oxygen parameters read by the Modbus protocol,the data is sent to GPRS mobile data transmission module for data transmission,at the same time,they are displayed in numerical and waveform by remote data acquisition server.The wireless data acquisition mode'construction and operation cost is low,it also has the advantages of wide monitoring range,high real-time performance,and the construction of NiosII microprocessor makes the system easy to upgrade,it can better meet the requirements of water environment monitoring.
\end{abstract}

\section{Introduction}

As an artificial reservoir,the Hongze lake is the water of local industrial and agricultural and people's living,once the water getting worse,the living of urban and rural residents will be seriously affected,therefore, the real-time monitoring of the Hongze lake'water quality is very important.At present,the traditional mean of domestic water environment monitoring[1] is mainly artificial field-sampling and instrumental analysis which is a tedious work,the sampling error is big,monitoring data scattered,and cannot guarantee the accuracy of data,the managers often get monitoring data which has been lagging behind,the main reason of the phenomenon is the data'acquisition process needs to be improved,on the other hand,the complicated Hongze Lake'geographical environment causes the wired network can not fully cover the area,and the monitored data cannot be real-time delivered.

Therefore,this paper puts forward a online data acquisition system using GPRS which can real-time or timing acquisition long-distance data.Relative to the wired transmission mode,the transmission rate of wireless is fast,and it has low construction and operation cost, wide monitoring range,high real time,the online water quality automatic monitoring system which using the data transmission form can ensure relevant departments timely get continuous monitoring data. 


\section{The hardware composition of system}

The system controlled by Nios II[2] can transfer wirelessly the culture water quality parameters (temperature, $\mathrm{PH}$ and dissolved oxygen) of the Hongze Lake detected by field sensor to remote monitoring center through the GPRS, the water quality monitoring center can display and storage three parameters and alarm when beyond the indicators. The design of the remote data transmission communication system is the focus of this topic,it complete the data transmission by using FPGA[3] as the main control chip and the Nios II microcomputer[5] construct by SOPC technology as controller.The overall structure of the system diagram as shown in Fig.1.

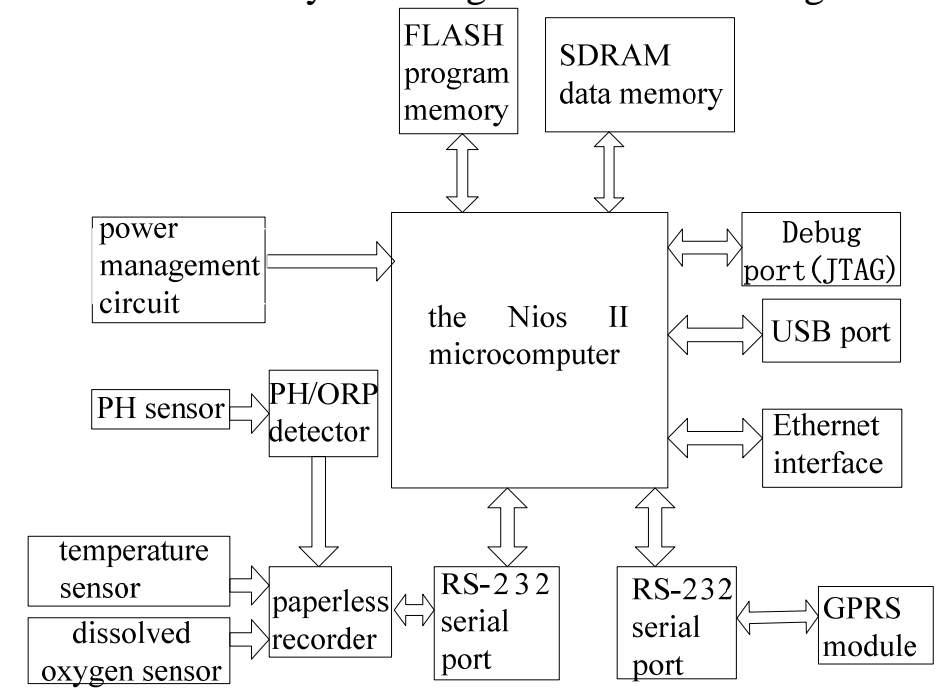

Fig. 1 The structure diagram of water quality monitoring station

From fig.1,you can see that the whole water quality monitoring system is composed of three water quality sensor( $\mathrm{PH}$ sensor, temperature sensor and oxygen sensor),a power management circuit,GPRS module(SIM300), the communication interface(debug port,USB port and Ethernet interface),memory module(FLASH program memory and SDRAM data memory) and Nios II processor[4],the input and output components etc.

When the system is powered on,PH sensor,temperature sensor and dissolved oxygen sensor began collecting information,among them,the electrical signal which $\mathrm{PH}$ sensor collected is sent to the paperless recorder after processed by the PH/ORP detector,the data collected by temperature and dissolved oxygen sensor is also transmitted to the paperless recorder,finally,the paperless recorder transmit the data to the main control CPU through serial port and Modbus protocol,then,the data will be sent to the control center by CPU and SIM300 serial module through the GPRS network.

The paperless recorder in the system can detect parameters of PH,temperature,dissolved oxygen and can set the alarm limit of upper and lower,it can verify the validity of the transmission data by comparing PC'received data with the local testing data.The paperless recorder can communicate with other devices for it's RS-485 serial port,therefore,after convert RS-485 to RS-232 through the converter,it is connectted with Nios II microprocessor by serial 0, and transfer the data to the Nios II microprocessor according to the Modbus protocol,in the end,it complete the wireless data transmission by SIM300 module.

Because the interface of module is RS232 protocol interface[5],it also need to design the UART interface circuit which meet the requirements of SIM300 wireless data transmission protocol between the Nios II and SIM300 using FPGA chip,the electrical connection between the two can be realized incombined with the RS232 protocol level conversion circuit.Finally,Nios II microprocessor communicate with the GPRS module through another RS-232 serial port,and SIM300 module send the data to the china mobile network through AT commands. 


\section{The realization of the hardware design}

\section{The construction of Nios II system}

The IP nuclear of system is constructed by SOPC Builder tool.Added NiosII processor,SDRA $M$ module,Avalon three state bridge,Flash memory interface(CFI) module,SYSTEM ID,JTAG UART,UART (RS-232) during the construction of system'IP nuclear,and realize the automatic allocation of address and interrupt through the connection of cfi_flash and three state bridge,and click on the SYSTEM->Auto-assign Basic Addresses and SYSTEM->Auto-assign IRQs.The construction of specific Nios II system as shown in Fig.2.

\begin{tabular}{|c|c|c|c|c|c|}
\hline Connecti... & Name & Description & Clock & Base & End \\
\hline & $\begin{array}{l}\text { 日 cpu } \\
\text { instruction_master } \\
\text { data_master } \\
\text { jtag_debug_module }\end{array}$ & $\begin{array}{l}\text { Nios II Processor } \\
\text { Avalon Memory Mapped Master } \\
\text { Avalon Memory Mapped Master } \\
\text { Avalon Memory Mapped Slave }\end{array}$ & $\begin{array}{l}{[\mathrm{clk}]} \\
\text { clk_0 } \\
{[\mathrm{ckl}]} \\
{[\mathrm{clk}]}\end{array}$ & $\begin{array}{r}\operatorname{IRQ} 0 \\
0 \mathrm{x} 00200800\end{array}$ & $0 x 00200$ fff \\
\hline & $\begin{array}{l}\text { 日 sdram } \\
\text { s1 }\end{array}$ & $\begin{array}{l}\text { SDRAM Controller } \\
\text { Avalon Memory Mapped Slave }\end{array}$ & $\begin{array}{l}{[\mathrm{clk}]} \\
\text { clk_0 }\end{array}$ & if $0 \mathrm{x} 01000000$ & 0x017ffffe \\
\hline & $\begin{array}{l}\text { 日 tri_state_bridge } \\
\text { avalon_slave } \\
\text { tristate_master }\end{array}$ & $\begin{array}{l}\text { Avalon-MM Tristate Bridge } \\
\text { Avalon Memory Mapped Slave } \\
\text { Avalon Memory Mapped Tristate Master }\end{array}$ & $\begin{array}{l}\text { clk_0 } \\
\text { [ckl] }\end{array}$ & & \\
\hline$\rightarrow$ & $\begin{array}{c}\boxminus \text { cfi_flash } \\
s 1\end{array}$ & $\begin{array}{l}\text { Flash Memory Interface (CFI) } \\
\text { Avalon Memory Mapped Tristate Slave }\end{array}$ & clk_0 & - $0 x 00000000$ & 0x001ffiff \\
\hline & $\begin{array}{l}\boxminus \text { sysid } \\
\text { control_slave }\end{array}$ & $\begin{array}{l}\text { System ID Peripheral } \\
\text { Avalon Memory Mapped Slave }\end{array}$ & $\begin{array}{l}{[\mathrm{clk}]} \\
\text { clk_0 }\end{array}$ & If $0 x 00201040$ & $0 x 00201047$ \\
\hline & $\begin{array}{l}\boxminus \text { jtag_uart } \\
\text { avalon_tag_slave }\end{array}$ & $\begin{array}{l}\text { JTAG UART } \\
\text { Avalon Memory Mapped Slave }\end{array}$ & $\begin{array}{l}{[\mathrm{clk}]} \\
\text { clk_0 }\end{array}$ & If $0 \mathrm{x} 00201048$ & $0 x 0020104 f$ \\
\hline 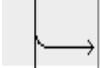 & $\begin{array}{l}\boxminus \text { uart_o } \\
s 1\end{array}$ & $\begin{array}{l}\text { UART (RS-232 Serial Port) } \\
\text { Avalon Memory Mapped Slave }\end{array}$ & $\begin{array}{l}{[\mathrm{clk}]} \\
\text { clk_0 }\end{array}$ & If $0 x 00201000$ & $0 z 0020101 \mathrm{f}$ \\
\hline & $\begin{array}{c}\boxminus \text { uart_1 } \\
s 1\end{array}$ & $\begin{array}{l}\text { UART (RS-232 Serial Port) } \\
\text { Avalon Memory Mapped Slave }\end{array}$ & $\begin{array}{l}{[\text { clk] }} \\
\text { clk_0 }\end{array}$ & If $0 \mathrm{x} 00201020$ & $0 x 0020103 f$ \\
\hline
\end{tabular}

Fig.2 The construction of Nios II system

\section{The construction of the SIM300 module'interface circuit}

In the design of the system,the SIM 300 module interface circuits is build on the same FPGA chip by the soft core.The construction is shown in Fig.3.The asynchronous serial port UART1 including RTS and CTS signal is constructed using UART soft core,the RXD,TXD of UART1 are connected respectively with the pins of SIM300 module'data receiving and sending,the RTS pin is used for transmitting the request signal of SIM300 module'data transmission and it will output low signal before sending data,the SIM300 module response via the CTS pin,so,the handshake of the data transmission is complete.

$\begin{array}{lr}\text { cts_n_to_the_uart_1 } & \text { rts_n_from_the_uart_1 } \\ \text { rxd_to_the_uart_1 } & \text { txd_from_the_uart_1 }\end{array}$

Fig.3 The construction of the SIM 300 module'interface circuits

\section{The construction of paperless recorder module'interface circuit}

The construction of the paperless recorder module'interface circuit as shown in Fig.4.It is also implemented in the same FPGA using soft core. The construction method are similar to SIM300 module interface circuit,no longer restatement.

$\begin{array}{lr}\text { cts_n_to_the_uart_0 } & \text { rts_n_from_the_uart_0 } \\ \text { rxd_to_the_uart_0 } & \text { txd_from_the_uart_0 }\end{array}$

Fig.4 The construction of the paperless recorder module'interface circuit

\section{The selection and circuit connection of sensor}

In this system,the dissolved oxygen sensor is YDC-100 which output signal is analog voltage 
signal,its output $\leqslant 0.3 \mathrm{mV}$ in aqueous solution of $25^{\circ} \mathrm{C}$ normal temperature which dissolved oxygen equal to zero.Temperature sensor using PT100 thermal resistance,PH sensor is PH-GP100PH which output is analog current.The three kinds of sensor are connected with the paperless recorder.The recorder has 3 universal input channels and 1 RS-232C communication interface, $\mathrm{V}+\mathrm{I}+$ and GND is one of the input terminals, wherein, $\mathrm{V}+$ is voltage signal input terminal,I+ is current signal input terminal,GND is ground terminal.In the system,the channel 1 is dissolved oxygen input channel,channel 2 is the temperature input channel,channel 3 is $\mathrm{PH}$ input channel.In the work process,the recorder communicate with Nios II microprocessor through the serial port 0 and Modbus protocol.The connection form of three sensors and recorders is similar,in cases of dissolved oxygen sensor,its connected diagram with paperless recorder as shown in Fig.5.

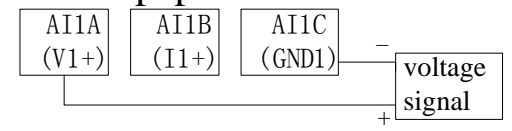

Fig. 5 the connection diagram of dissolved oxygen sensor with paperless recorder

\section{The software design}

The software part mainly includes the main program,SIM300 module communication program and sensor 'writing and reading program.

\section{The main program design}

The main program include interrupt enable program,CRC check program,timer 1 interrupt program and the main function.First,it call two serial port initialization program,and detect the flag of ReceiveGprs which indicates that the end of data receiving while it is 0xff,then set the Modbus protocol frame by changing the top eight of the command array,so as to read the dissolved oxygen,temperature and $\mathrm{PH}$ data,then,it determine the error cause and error types by the returning command[1] in the reading sensor subroutine.In the design,the sensor information is read through the interrupt,serial port interrupt request flag of receiving or sending plays an important role. When Nios receiving or sending a frame data,the flag is set 1 by hardware which request to the CPU'interrupt,when CPU turned to the interrupt service program,the bit must be cleared by software.In the design of CRC check,according to the Modbus based on serial port,using the station address of 1 byte additional address field,and 2 byte CRC check of data verification domain,the error detection fields is derived through the method of the lengthy cycle.

\section{The initialization program design of GPRS module SIM300}

SIM300 module'initialization begins automatically after the system power and complete the serial initialization,GPRS module SIM300 realize communication with Nios through the serial port 1.The main parameters of GPRS initialization process is the CurrentFlag value which represents the current state.First,the program judge whether the CTS effectively,and CurrentFlag will equal to 0x00 if it is invalid,then, The Nios can send initialization instructions to GPRS which completed by calling a serial sending subroutine and a strcpy function and the Command array,the judge of response is complete by using the StrCmp function and the Command array,next,set the short message to the text transmit mode,and add head marking to GPRS data,connect to the server, if the connection is successful for the first time,the flag of Current returns 0x09, if not,it close the TCP connection and Current flag returns 0x05.

\section{The sensor reading program design}

By repeatedly calling the receiving subroutine of serial port 0 to achieve sensor'reading operation,sensor reading program has been called in the main function,and determined corresponding operation process by its returning CMD,CMD is the function code of Command[1] 
in the Modbus protocol which indicate that received data completed if it returns 0,and conversely,it indicate that received data error.

\section{Summary}

The environment online data acquisition system use SOPC technology to build a NiosII processor in the FPGA, and design the NiosII control program according to the SIM300 wireless data transmission,design the corresponding data transceiver,module initialization procedures and data application layer protocol aiming at SIM300 module'features,so as to realize the remote data transmission function of SIM300 module.The system provides a general implementation method for other remote data transmission which adopted NIOS II processor as the main control chip of the system,it simplifies the design process,improves the system'performance and development efficiency,at the same time,it is easy to upgrade.

\section{Reference}

[1]CaiQiang,HeMiao.Research and development of electrochem ical biosensor system for automatic waterborne pollutant analysis[J].Chinese Journal of Scientific Instrument, 2007:28(12):2158-2162

[2]ZOU Yu,WANG Cheng.Nios II processor-based system design multi-function counter[J].Manufacturing automation, 2011:33(8):146-149

[3]Yiannacouras,P.Steffan,J.G.Rose.Exploration and Customization of FPGA-Based Soft Processors[J].Computer-Aided Design of Integrated Circuits and Systems, 2007:26(2): 266-277

[4]Hu Jisheng,Li Hong.Arbitrary waveform generator design and realization based on SoPC/NIOS II[J].The application of electronic technology,2011:37(6):91-94

[5]Ouyang Danna,Li Yinghong.Research on Communication between RS-232 and PROFIBUS[J].Chinese Journal of scientific instrument,2005:26(8):82-89 